\title{
Management of a permanent maxillary first molar with two disto buccal canals with the aid of spiral computed tomography: a case report
}

\author{
Anand C Patil ${ }^{1}$, Ramesh H G ${ }^{2}$, Sandhya Yelamali ${ }^{3}$ \\ ${ }^{1}$ Professor. Department of Conservative Dentistry and Endodontics, KLE VK Institute of Dental Sciences, Belgaum, Karnataka, \\ India. \\ ${ }^{2}$ Reader. Department of Conservative Dentistry and Endodontics, KLE VK Institute of Dental Sciences, Belgaum, Karnataka, \\ India. \\ 3 PG student. Department of Conservative Dentistry and Endodontics, KLE VK Institute of Dental Sciences, Belgaum, Karna- \\ taka, India.
}

\author{
Correspondence: \\ Dr Anand C Patil, \\ Department of Conservative Dentistry and Endodontics, \\ KLE VK Institute of Dental Sciences, Nehru Nagar, \\ Belgaum, 590010 \\ Karnataka, INDIA \\ dranandp@gmail.com
}

\begin{abstract}
Human molars show considerable anatomic variations with respect to number of roots and root canals. The most common form of the permanent maxillary first molar has three roots and four canals, two mesiobuccal canals and one distobuccal and palatal canal each. $98.3 \%$ of maxillary first molar have been reported to have a single distobuccal canal. Two canals were found only $1.7 \%$ of the time, hence showing the second disto buccal canal to being a rare occurrence.

Conventionally radiographs are used to determine root canal anatomy, which is a 2- dimensional representation of a 3-dimensional object. Diagnostic methods like spiral computerized tomography where a 3 dimensional data is acquired are useful to determine complex morphology. This case report presents the management of a maxillary first molar with two disto buccal root canals confirmed with the aid of computed tomography along with the conventional radiographic method.

Computed Tomography scan slices revealed two separate distobuccal canals at the coronal 3rd of the root. The second distobuccal canal was missing in the middle and apical 3rd indicating; it might have joined the distobuccal canal at the middle $3 \mathrm{rd}$ area.
\end{abstract}

Key words: Maxillary molars, Root canal anatomy, Spiral Computed Tomography. 


\section{Introduction}

Thorough cleaning and shaping of all the pulp spaces and its complete filling with an inert material is one of the main objectives of root canal treatment (1). In routine endodontic practice, clinicians have to treat teeth with atypical configurations. Hence, a thorough knowledge of the external and internal anatomy of teeth is an important aspect of root canal treatment (2). Root canals might be left untreated, if the dentist fails to identify the presence of atypical configurations such as extra roots/ root canals (3).

Various studies have demonstrated substantial variation in human maxillary molar anatomy regarding the number of roots and root canals (2). The most common form of the permanent maxillary first molar has three roots and four canals with majority of the mesiobuccal roots having two canals while distobuccal and palatal roots having a single canal. The mesiobuccal root of the first maxillary molar has generated more research and clinical investigation than any other root (4). $93.3 \%$ of maxillary first molar have been reported to have a single distobuccal canal. The presence of a second distobuccal canal in maxillary first molars has been reported to be present as low as $1.7 \%$, and hence occurrence of a second distobuccal canal is rare (4).

Such complex root canal anatomies have been conventionally diagnosed with radiographs, using different angulations which provide sufficient information to the clinician. But due to problems like overlap between the roots and the superimpositioning of the anatomical structures on the tooth, the radiographs can sometimes prove inconclusive and do not provide detailed information concerning the 3-dimensional image, which would help the clinician in making a confirmatory diagnosis (5). Newer techniques like spiral computed tomography (CT) are being used to study root canal morphology as a 3D image.

This case report describes the detection and management of a permanent maxillary first molar with a rare occurrence of two distobuccal canals with the aid of spiral computed tomography.

\section{Case Report}

A healthy 56 year old male patient presented to the Department of Conservative Dentistry and Endodontics, KLE VK Institute of Dental Sciences Belgaum, Karnataka, with the complaint of pain in the upper right back tooth since one week. The pain was continuous in nature and increased on mastication, food lodgment and on having sweets. On clinical examination, a deep carious lesion was found in the right maxillary first molar. The tooth was tender to percussion. Radiographic examination of the tooth revealed a large carious lesion that was seen encroaching onto the pulp space. Thickening of the periodontal space was also seen (Fig. 1a). Hence, a

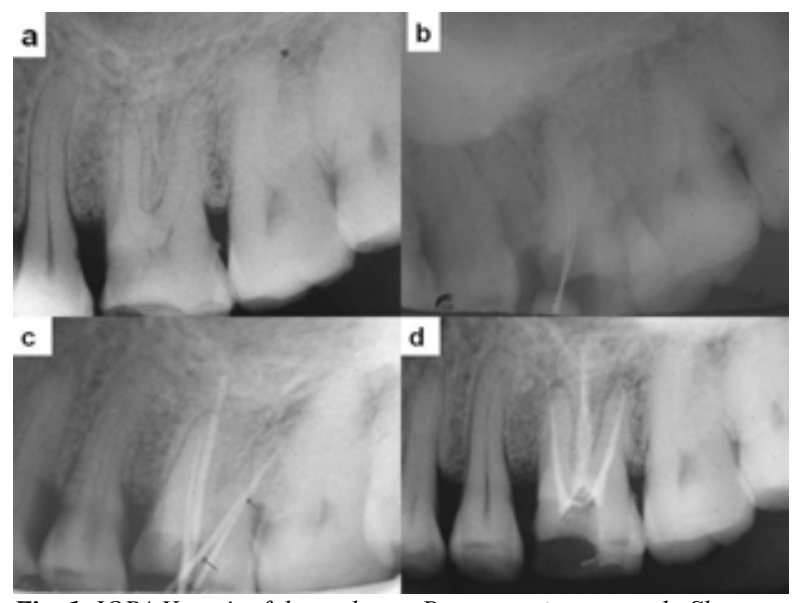

Fig. 1. IOPA X-ray's of the molar. a. Pre-operative $x$ ray; $b$. Shows the files in the two distobuccal canals; $c$. Shows Master cones in the two distobuccal canals; $d$. Post-obturation $x$-ray.

diagnosis of acute irreversible pulpitis was made and the tooth was prepared for endodontic therapy. His medical history was noncontributory.

After local anesthesia, a conventional endodontic access opening was made. While the access chamber was being explored with a DG 16 explorer (Maillefer, Dentsply), a catch was felt between the line joining the distobuccal and the palatal canals, more closely towards the distobuccal canal. The presence of an extra root/ root canal or a perforation was suspected (Fig. 2). A No $10 \mathrm{~K}$ file (Mani Inc, Japan) was placed in the suspected canal and a conventional radiograph was taken at different horizontal angulations (Fig. 1b). The instrument caused no bleeding or pain and reached a similar length as that of the distobuccal canal. Due to the overlap between the distobuccal canals and the superimposition of the anatomical structures on the distobuccal root, the radiograph proved inconclusive. Hence, to confirm whether the second canal was truly in the distobuccal root, a spiral $\mathrm{CT}$ was used. The patient was prescribed analgesics and discharged after sealing the cavity with a sterile cotton pellet and Caviton (GC Corporation Tokyo, Japan).

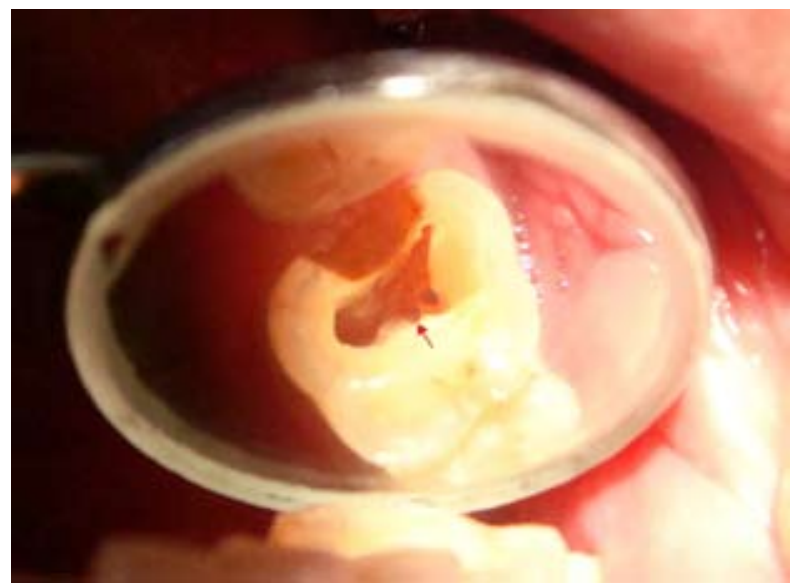

Fig. 2. Intra oral view showing the two distobuccal canals e154 
The patient was recalled back the next day and the tooth was scanned using a multi-detector CT scanner (Sensation 64, Siemens, Germany) at the Department of Radiology, KLE's Prabhakar Kore Hospital and Research Centre, Belgaum using the following parameters, 64 slices $/ \mathrm{sec}$, collimation $0.6 \mathrm{~mm}$, pitch 0.9 , tube voltage $120 \mathrm{kV}$, and tube current $390 \mathrm{~mA}$ was used. The scan reconstruction interval was $0.6 \mathrm{~mm}$ resulting in 200-240 overlapping images. Axial images were transmitted to an inbuilt dental program.

Horizontal slices of the molar were studied at different levels (coronal, middle and apical 3rd of the root) to determine the canal morphology. CT scan slices revealed two separate distobuccal canals at the coronal 3rd of the root (Figs. 3a and 3b). The second distobuccal canal was missing in the middle and apical 3rd (Figs. 3c and 3d), indicating it might have joined the distobuccal canal at the middle 3 rd area.

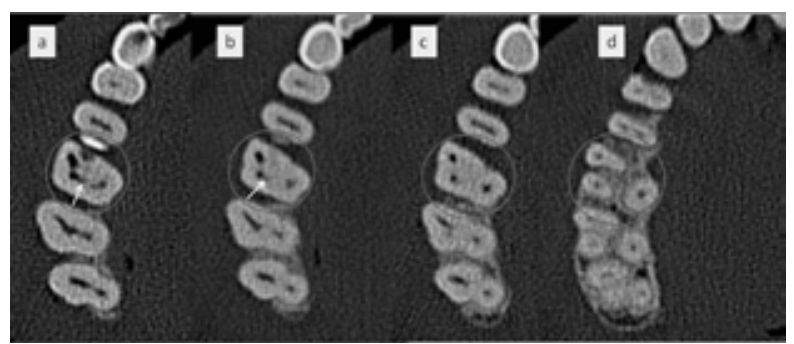

Fig. 3. Spiral CT images; a. Coronal third; b.Middle third; $c \& d$. apical third.

At the next visit, working length of each of the canals was estimated using an electronic apex locator (Raypex 5, VDW Endodontic synergy, Germany) and then confirmed by a radiograph. During the estimation of the working length, when the instruments were placed in both the distobuccal canals, one of the instruments was hindered by the other (in the middle 3rd area) in reaching the working length, reconfirming the fact that the two canals were joining in the middle 3rd area. All the canals were coronally flared using Gates Glidden drills (Mani Inc, Japan). Cleaning and shaping was performed with a step back technique using stainless steel $\mathrm{K}$ files (Mani Inc, Japan) and a copious irrigation of $3 \% \mathrm{NaOCl}$ (Vishal Dentocare Pvt Ltd, Sarkhej, Ahmedabad). The buccal canals were prepared to ISO size 35 and the palatal canal to size 40 respectively. The canals were obturated by lateral condensation technique using gutta percha and a zinc oxide eugenol sealer (Figs. 1c and 1d), and the tooth was then restored with a composite resin (Filtek P60, 3M/ESPE).

\section{Discussion}

Anatomical variations are not uncommon in molars (6). The permanent maxillary first molar commonly has three roots and four canals with majority of the mesiobuccal roots having two canals while distobuccal and palatal roots having a single canal (4). Studies on maxillary first molar have reported the most common canal system morphology of the distobuccal root was a single canal $(93.3 \%)$. Two canals were found only $1.7 \%$ of the time of which a single apical foramen was present $98 \%$ of the time, hence showing the second disto buccal canal to being a rare occurrence (4). 2 or more distobuccal canals were reported in about $9.50 \%$ of the teeth studied of which $97 \%$ ended into a single apex (4). Another study reported 2 distobuccal canals in $1.90 \%$ of the cases (7). In yet another study $1.64 \%$ of cases had two distobuccal canals of which $98.40 \%$ of the cases ended into a single canal at the apex (8). It can be concluded form these reports that the incidence of two distobuccal canals in the maxillary first molar ranges from $1.64 \%-9.5 \%$, making it a rarity.

Every individual root has a unique canal system. It may have one root canal exiting in one apical foramen (type I), two root canals that join short of the apex (type II), two distinct canals emerging on the root surface through two distinct foramina (type III) or one root canal that bifurcates inside the root ending in two foramina (type IV) (2). This report presents a variation of maxillary first molar having two distobuccal root canals having a type II configuration which was confirmed with the help of spiral CT.

Periapical radiographs taken in different angulations are an essential part of endodontic treatment; however they are taken in a buccal-lingual direction and give only 2-dimensional information about a 3 -dimensional object. In recent years, advanced techniques like CT are being used to evaluate root canal morphology as a 3D image. Conventional CT uses X-rays to generate cross-sectional (axial) images. The X-ray beam is swept around the object; after passing through it, the radiation strikes a series of detectors that either move with X-ray tube or are stationary. Relatively advanced mathematical techniques are used to create a 2D image of the crosssectional volume that is scanned (9).

Spiral CT is a recent advance in CT technology (9). It has proved to be a useful tool in diagnosing complex root canal anatomies. By using simultaneous patient translation through the $\mathrm{x}$ ray source with continuous rotation of the source-detector assembly, spiral computed tomography acquires raw production data with a spiral sampling locus during a relatively short period. Without any additional scanning time, these data can be viewed as conventional transaxial images such as multiplaner reconstructions or as 3-dimensional reconstructions (5). Contiguous $1 \mathrm{~mm}$ thick transaxial images of the tooth can be obtained and there is no possibility for missing any dental structures. Images can be easily reconstructed into different planes (9).

Spiral CT offers a more rapid examination time and with an effective dose much less than the conventional CT 
(5). The only disadvantage with the spiral CT technique can be its cost effectiveness for the patient. The CT scan would be relatively more expensive than a conventional radiograph for the patient.

Variations in the root canal morphology of the maxillary first molar are quiet common. During endodontic therapy if the practitioner is unable to detect these variations the root canals might be left untreated which may be a reason for the failure of the endodontic treatment. Thus a clinician should be aware of such variations and should open up his/her eyes to newer diagnostic techniques like spiral CT that would help in the detection of such abnormalities and help in the eventual success of endodontic therapy.

\section{References}

1.European Society of Endodontology. Quality guidelines for endodontic treatment: consensus report of the European society of Endodntology. Int Endod J 2006; 39, 921-30

2.Fava L R G. Root canal treatment in an unusual maxillary first molar: a case report. Int Endod J 2001; 34: 649-53.

3.Aggarwal V, Singla M, Logani A, Shah N. Endodontic management of a maxillary first molar with two palatal canals with the aid of spiral computed tomography: a case report. J Endod 2009; 35: 137-39.

4.Cleghorn BM, Christie WH, Dong CCS. Root and root canal morphology of the human permanent maxillary first molar: a literature review. J Endod 2006; 32: 813-21.

5.Reddy YP, Karpagavinayagam K, Subbarao CV. Management of dens invaginatus diagnosed by spiral computed tomography: a case report. J Endod 2008; 34: 1138-42.

6.Hulsmann M. A maxillary first molar with two disto-buccal root canals. J Endod 1997; 23: 707-8.

7.Alavi AM, Opasanon A, Ng YL, Gulabivala K. Root and canal morphology of Thai maxillary molars. Int Endod J 2002; 35: 478-85.

8.Caliskan MK, Pehlivan Y, Sepetcioglu F, Turkun M, Tuncer SS. Root canal morphology of human permanent teeth in a Turkish population. $\mathrm{J}$ Endod 1995; 21: 200-4.

9.Cimilli H, Cimilli T, Mumcu G, Kartal N, Wesselink P. Spiral computed tomographic demonstration of $\mathrm{C}$-shaped canals in mandibular second molars. Dentomaxillofac Radiol 2005; 34: 164-67. 